\title{
Clusterin inhibition to enhance tumor chemosensitivity in systemic tumors
}

\author{
Shailendra Kapoor
}

Received: 3 October 2012/ Accepted: 31 December 2012/Published online: 6 February 2013

(C) Springer-Verlag Berlin Heidelberg 2013

To the editor

I read with great interest the recent article by Cheng et al. [1]. Interestingly, inhibition of clusterin activity enhances the sensitivity to a number of other chemo-therapeutic agents in a number of other systemic malignancies besides lung carcinomas.

For instance, OGX-011 can be used to inhibit clusterin in renal cell carcinomas. This inhibition of clusterin enhances the sensitivity to and the anti-neoplastic effects of sorafenib [2]. The combination exerts marked synergism in decreasing proliferation in renal cell malignancies by virtue of significant down-regulation of phosphorylated Akt. Similarly, the use of antisense-oligo deoxynucleotides to inhibit clusterin accentuates the radio-sensitivity of bladder carcinomas [3]. Similarly, antisense oligonucleotides against clusterin have been shown to increase radiochemosensitivity in prostate carcinomas [4]. The efficacy of androgen ablation is also accentuated following treatment with these antisense oligonucleotides.

Similarly, accentuated clusterin expression usually indicates a poor response to radiotherapy as well as chemotherapy in breast cancers. Down-regulation of clusterin increases the sensitivity to tamoxifen in ER anti-estrogen resistant breast cancer cells [5]. Similarly, in ovarian carcinomas, down-regulation of clusterin increases sensitivity

This comment refers to the article available at doi:10.1007/s00280-011-1682-0.

An author's reply to this comment is available at doi:10.1007/s00280-013-2085-1.

S. Kapoor $(\bowtie)$

Private Practise, Mechanicsville, VA, USA

e-mail: shailendrakapoor@yahoo.com to agents such as paclitaxel [6]. The down-regulation can be achieved by transfection of siRNA or by using OGX011. This results in improved prognosis and a better clinical outcome in these tumors.

The above examples clearly illustrate the efficacy of clusterin targeting in increasing radio as well as chemosensitivity in a number of systemic tumors. Clearly, there is a need to develop further anti-clusterin therapy that may very well change the management of chemoresistamt malignancies in the near future.

\section{References}

1. Cheng CY, Cherng SH, Wu WJ et al (2012) Regulation of chemosensitivity and migration by clusterin in non-small cell lung cancer cells. Cancer Chemother Pharmacol 69:145-154

2. Kususda Y, Miyake H, Gleave ME, Fujisawa M (2012) Clusterin inhibition using OGX-011 synergistically enhances antitumour activity of sorafenib in a human renal cell carcinoma model. Br J Cancer 106:1945-1952

3. Yamanaka K, Gleave M, Muramaki M, Hara I, Miyake H (2005) Enhanced radiosensitivity by inhibition of the anti-apoptotic gene clusterin using antisense oligodeoxynucleotide in a human bladder cancer model. Oncol Rep 13:885-890

4. Gleave M, Miyake H (2005) Use of antisense oligonucleotides targeting the cytoprotective gene, clusterin, to enhance androgenand chemo-sensitivity in prostate cancer. World J Urol 23:38-46

5. Cappelletti V, Gariboldi M, De Cecco L et al (2008) Patterns and changes in gene expression following neo-adjuvant anti-estrogen treatment in estrogen receptor-positive breast cancer. Endocr Relat Cancer 15:439-449

6. Hassan MK, Watari H, Han Y et al (2011) Clusterin is a potential molecular predictor for ovarian cancer patient's survival: targeting clusterin improves response to paclitaxel. J Exp Clin Cancer Res 30:113 